\title{
Oficina de saúde e sexualidade: Residentes de saúde promovendo educação sexual entre adolescentes de escola pública
}

\author{
Workshop on health and sexuality: Health residents promoting sexual education among \\ adolescents from a public school
}

\section{Oficina de salud y sexualidad: Residentes de salud promoviendo educación sexual entre adolescentes de escuela pública}

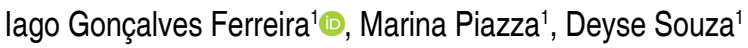 \\ ${ }^{1}$ Escola de Saúde Pública de Florianópolis. Florianópolis, SC, Brasil.
}

\section{Resumo}

Objetivo: Relatar a experiência da implementação de oficinas de saúde e sexualidade por residentes de saúde da família com adolescentes do $8^{\circ}$ ano de uma escola pública em Florianópolis. Relato de experiência: As oficinas foram organizadas sob a forma de encontros mensais, com turmas do 8o ano dos períodos matutino e vespertino, da Escola de Ensino Básico Hilda Teodoro, situada no bairro da Trindade, em Florianópolis, entre os meses de março a dezembro de 2017. Os encontros eram realizados em pequenos grupos, com 16 alunos por turma, dispostos em roda de maneira a permitir a interação e participação de todos os membros, tendo duração de 45 a 90 minutos. Durante os encontros, eram realizadas palestras, dinâmicas e rodas de conversa, estimulando a reflexão, debate e conscientização dos adolescentes acerca da importância de temas como: gravidez na adolescência, doenças sexualmente transmissíveis, orientação sexual e identidade de gênero, machismo, métodos anticoncepcionais e anatomia dos órgãos reprodutivos. Discussão: Por meio da experiência, notou-se participação ativa e ricas contribuições por parte dos adolescentes, principalmente das meninas, evidenciando interesse considerável sobre a temática da sexualidade, principalmente a gravidez na adolescência e os métodos contraceptivos. Os discursos e pontos de vista ressaltaram as mudanças em curso na sociedade brasileira, em direção à igualdade de gênero e empoderamento feminino. Conclusão: As oficinas de saúde e sexualidade desenvolvidas pelos residentes de medicina de família e comunidade e saúde da família representaram uma oportunidade ímpar de interação entre os programas de residência, as equipes de Estratégia de Saúde da Família e a comunidade, utilizando o cenário escolar como ferramenta para a promoção de saúde e empoderamento social.

Palavras-chave: Especialização; Internato e Residência; Sexualidade; Adolescente

Como citar: Ferreira IG, Piazza M, Souza D. Oficina de saúde e sexualidade: Residentes de saúde promovendo educação sexual entre adolescentes de escola pública. Rev Bras Med Fam Comunidade. 2019;14(41):1788. http://dx.doi.org/10.5712/rbmfc14(41)1788

Autor correspondente: lago Gonçalves Ferreira. E-mail: iago_goncalves14@hotmail.com Fonte de financiamento: declaram não haver. Parecer CEP: não se aplica. Procedência e revisão por pares: revisado por pares. Recebido em: 28/06/2018. Aprovado em: 22/01/2019. 


\begin{abstract}
Objective: To describe an experience of implementing a health and sexuality workshop by family health residents with students from $8^{\text {th }}$ grade classes from a public school in Florianópolis. Experience report:Workshops were organized in the form of monthly meetings, with $8^{\text {th }}$ grade classes of morning and afternoon shifts, from the Hilda Teodoro Elementary School, located in the Trindade neighborhood, Florianópolis, from March to December, 2017. The meetings were performed in small groups, with 16 students per class, organized in circle allowing the interaction and participation of all members, lasting from 45 to 90 minutes. During the workshops, lectures, dynamics and conversation were developed, stimulating adolescents to wonder, discuss and reflect about the importance of topics such as teenage pregnancy, sexually transmitted diseases, sexual orientation and gender identity, machismo, contraceptive methods and anatomy of the reproductive organs. Discussion: Through the experience, it was noticed active participation and rich contributions by the adolescents, mainly of the girls, evidencing considerable interest on the subject of sexuality, especially teenage pregnancy and contraceptive methods. The speeches and points of view highlighted the changes taking place in Brazilian society, towards gender equality and women's empowerment. Conclusion: Health and sexuality workshops organized by family medicine and family health residents represented a relevant opportunity to interaction amongst Family Health Strategy Residence Programs, Family Health Strategy teams and communities, using the school scenario as a tool for health promotion and social empowerment.
\end{abstract}

Keywords: Specialization; Internship and Residency; Sexuality; Adolescent

\title{
Resumen
}

Objetivo: Informar la experiencia de la implementación de talleres de salud y sexualidad por residentes de salud de la familia con adolescentes del 8 año de una escuela pública en Florianópolis. Relato de experiencia: Los talleres fueron organizados en forma de encuentros mensuales, con clases del $8^{\circ}$ año, períodos matutino y vespertino, de la Escuela de Educación Básica Hilda Teodoro, ubicada en el barrio de la Trinidad en Florianópolis, entre los meses de marzo a diciembre de 2017. Los encuentros se realizaban en pequeños grupos, con 16 alumnos por clase, dispuestos en de manera que permita la interacción y participación de todos los miembros, teniendo una duración de 45 a 90 minutos. Durante los encuentros se realizaron charlas, dinámicas y ruedas de conversación, estimulando la reflexión, debate y concientización de los adolescentes acerca de la importancia de temas como embarazo en la adolescencia, enfermedades sexualmente transmisibles, orientación sexual e identidad de género, machismo, métodos anticonceptivos y anatomía de los adolescentes órganos reproductivos. Discusión: Por medio de la experiencia, se notó participación activa y ricas contribuciones por parte de los adolescentes, principalmente de las niñas, evidenciando un interés considerable sobre la temática de la sexualidad, principalmente el embarazo en la adolescencia y los métodos anticonceptivos. Los discursos y puntos de vista resaltaron los cambios en curso en la sociedad brasileña, en dirección a la igualdad de género y empoderamiento femenino. Conclusión: Los talleres de salud y sexualidad desarrollados por los residentes de medicina de la familia y salud de la familia representaron una oportunidad relevante de interacción entre los Programas de Residencia en Salud de la Familia, los equipos de Estrategia de Salud de la Familia y la comunidad, utilizando el escenario escolar como herramienta para la promoción de la salud y el empoderamiento social.

Palabras clave: Especialización; Internado y Residencia; Sexualidad; Adolescente

\section{Introdução}

A adolescência consiste em um período crítico de transição entre a infância e a vida adulta. Nessa complexa fase, os adolescentes sofrem intensas transformações corporais, cognitivas e comportamentais, que propiciam novas descobertas e experiências que contribuirão para a construção de sua identidade e autonomia. ${ }^{1-3}$

Durante o processo de amadurecimento, os adolescentes apresentam comportamentos extremos, demonstrando certa negligência com a própria saúde. Tal aspecto tem sido reforçado pelas mudanças no comportamento sexual dos jovens nos últimos anos, com início precoce da atividade sexual, aumento do número de parceiros e de casos de doenças sexualmente transmissíveis, incluindo o HIV. ${ }^{4-6}$

Dessa maneira, torna-se evidente a importância da discussão da sexualidade, visto que esta ainda se apresenta como um tabu social, cercada de mitos, estereótipos, valores e crenças, restringindo-se a diálogos superficiais entre pais e filhos ou à abordagem tradicionalmente 'biologicista' do currículo escolar. ${ }^{7-9}$ $\mathrm{O}$ acesso dos jovens a fontes de informações discutíveis como amigos, internet e revistas, contribui para a carência de orientações consistentes, que acrescida da inserção limitada aos serviços de atenção primária à saúde, favorece atitudes de risco, que tornam essa população mais vulnerável. ${ }^{2,8,9}$ 
Nessa perspectiva, a escola apresenta-se como espaço privilegiado para ações de promoção de saúde e educação sexual, devido a seu caráter formativo que pode servir de estímulo à mudança de comportamentos e hábitos dos adolescentes. ${ }^{10,11}$ Considerando-se esse cenário estratégico de atuação, os ministérios da Saúde e da Educação instituíram o Programa Saúde na Escola (PSE) em 2007, objetivando fortalecer o enfretamento de vulnerabilidades da população jovem. Por meio de articulação intersetorial, o programa propicia atenção integral a crianças e adolescentes da rede pública de ensino, desenvolvendo ações de promoção, atenção e prevenção em saúde.4,10,12

Na articulação de suas ações, o PSE tem a Estratégia de Saúde da Família (ESF) como parceira, de forma a estimular que as equipes ESF atuem nas escolas de seus territórios de adscrição, atendendo às demandas da comunidade escolar e integrando-se aos projetos político-pedagógicos das instituições. . $^{4,10}$ Dessa maneira, a abordagem por profissionais de saúde de temáticas intrínsecas à adolescência, como a sexualidade, pode desenvolver a autonomia e responsabilização pessoal dos jovens, contribuindo para o fortalecimento do vínculo com as equipes. ${ }^{13,14}$

Considerando o papel estratégico da ESF na consolidação do PSE, torna-se essencial a formação de profissionais de saúde capacitados a desenvolver atividades voltadas às comunidades. No entanto, as graduações em saúde ainda apresentam modelos pedagógicos que privilegiam a abordagem biomédica e o âmbito hospitalar, resultando em falta de integração entre os profissionais de saúde e as populações, prejudicando a compreensão dos determinantes sociais do processo saúde-doença. ${ }^{13,15}$

Nesse sentido, os Programas de Residência Médica e Multiprofissional em Saúde demonstram potencial para impulsionar a mudança de paradigma na formação dos profissionais, contribuindo para a superação da fragmentação do cuidado e promovendo a educação interprofissional, por meio de formação conjunta e aprendizagens compartilhadas, em consonância com as diretrizes do SUS e da ESF. ${ }^{16}$

Dessa maneira, esse artigo teve como objetivo descrever experiência acerca de atividades desenvolvidas em oficinas sobre saúde e sexualidade com alunos do $8^{\circ}$ ano da Escola de Ensino Básico Hilda Teodoro, localizada no bairro da Trindade, em Florianópolis. A intervenção teve como organizadores os residentes dos programas de Residência Médica em Medicina de Família e Comunidade e Residência Multiprofissional em Saúde da Família da Escola de Saúde Pública de Florianópolis, no período de março a dezembro de 2017.

\section{Relato de experiência}

\section{Contexto e cenário da experiência}

O Programa de Residência Médica em Medicina de Família e Comunidade deu início às suas atividades em 2002, em uma iniciativa do Departamento de Saúde Pública da Universidade Federal de Santa Catarina, a qual esteve vinculado até 2014, ano em que foi incorporado pela Secretaria Municipal de Saúde de Florianópolis. O Programa de Residência Multiprofissional em Saúde Família surgiu em 2015, por meio de parceria entre a Universidade do Estado de Santa Catarina (UDESC) e a Secretaria Municipal de Saúde de Florianópolis. Atualmente, ambos os programas estão vinculados à Escola de Saúde Pública de Florianópolis, departamento da Secretaria Municipal de Florianópolis. 
O bairro da Trindade situa-se na região central da cidade de Florianópolis, sendo composto por estrutura econômica e social diversificada, entremeando áreas de classe média alta e de maior vulnerabilidade social, como o Morro da Penitenciária, situado próximo à Escola Hilda Teodoro. O Centro de Saúde Trindade, homônimo ao bairro, é constituído por cinco equipes da Estratégia de Saúde da Família (ESF), sendo a unidade de saúde responsável pela atenção primária no bairro.

A articulação entre a Escola Hilda Teodoro e o Centro de Saúde Trindade ocorreu por meio de reuniões mensais previamente agendadas, destinadas às discussões de demandas levantadas pela escola. As reuniões eram nomeadas de "Grupo de Escuta", em que profissionais do centro de saúde acolhiam as demandas escolares, de forma a traçar estratégias para o atendimento integral e ação junto à comunidade adscrita. Por meio desses encontros mensais, surgiram também os temas a serem abordados nas oficinas do Programa Saúde na Escola.

\section{Delineamento e organização das oficinas}

O modelo adotado para a abordagem dos adolescentes foi a oficina, tendo em vista que tal modelo apresenta proposta de aprendizagem compartilhada, por meio de dinâmicas e discussões em grupo. Essas interações permitem um debate amplo com trocas de ideias e valores, possibilitando a autorreflexão e adoção de posturas mais críticas frente aos assuntos abordados. ${ }^{5,17}$

As oficinas foram organizadas sob a forma de encontros mensais, com turmas do $8^{\circ}$ ano dos períodos matutino e vespertino, entre os meses de março a dezembro de 2017. Os encontros eram realizados em pequenos grupos com 16 alunos por turma, tendo duração de 45 a 90 minutos, com os estudantes dispostos em roda de maneira a permitir as falas e as contribuições de todos.

De acordo com Martins et al., ${ }^{5}$ o número reduzido de integrantes em grupos de discussão pode favorecer a comunicação e o estabelecimento de vínculo de confiança entre adolescentes e profissionais, de maneira que estes não se sintam expostos e/ou desconfortáveis. O vínculo e a comunicação consistem em importantes ferramentas na interação e liberdade de expressão dos jovens, permitindo a exposição de opiniões, perspectivas e autocríticas, de forma a construir conhecimentos coletivamente. ${ }^{14}$

As oficinas adotaram temas que contemplavam as propostas das Diretrizes Nacionais para a Atenção Integral à Saúde de Adolescentes e Jovens na Promoção, Proteção e Recuperação da Saúde, lançadas pelo Ministério da Saúde em 2010, com objetivo de nortear as ações de saúde frente aos desafios da atual conjuntura da juventude brasileira. ${ }^{6}$

A educação sexual, nos padrões modernos, não deve restringir-se à abordagem de DSTs e métodos contraceptivos, ao contrário, deve incluir aspectos comportamentais, psicológicos e culturais, capacitando os indivíduos a tomar decisões conscientes e a exercer seus direitos sexuais e reprodutivos. , $^{2,7}$

Nesse sentido, os temas adotados nos encontros foram:

1. A cultura do machismo

2. Anatomia e fisiologia dos sistemas reprodutores masculino e feminino

3. Doenças sexualmente transmissíveis (DSTs)

4. Gravidez na adolescência

5. Relações e conflitos familiares 


\section{Métodos contraceptivos}

7. Orientação sexual e identidade de gênero

8. Projeto de vida

A abordagem desses assuntos desenvolveu-se por meio de rodas de conversa com os estudantes, tendo os residentes como facilitadores, fomentando discussões e problematizando os temas.

As rodas de conversa eram iniciadas com recursos lúdicos como apresentação de slides, cartazes, modelos e vídeos, sendo que estes últimos consistem em valiosas ferramentas para atrair a atenção dos adolescentes, devido ao seu efeito provocativo, recorrendo a analogias e contextos reais. Neste cenário, o facilitador tem o papel de conduzir o grupo de forma participativa e dinâmica, instigando a participação dos membros de maneira organizada..$^{5,18,19}$

\section{Atividades e temas: a sexualidade por diferentes ângulos}

Durante o primeiro encontro com os estudantes, os residentes apresentaram a proposta e os objetivos das oficinas. Em seguida, foi exibido o vídeo "Era uma vez outra Maria”, animação sem diálogos desenvolvida pelo Instituto Promundo, na qual são abordados temas como estereótipos de gênero, violência sexual e gravidez na adolescência. Logo após, em roda de conversa, foi realizada discussão acerca das situações apresentadas na animação, permitindo aos adolescentes expor suas opiniões, conceitos e dúvidas. Ao término da discussão, propôs-se a atividade "caixa de perguntas", na qual os adolescentes deveriam escrever em pedaços de papel suas dúvidas e curiosidades acerca do tema, de maneira anônima.

No segundo encontro, os residentes explanaram sobre a anatomia e fisiologia do sistema reprodutores masculino e feminino e acerca dos aspectos fisiológicos das relações sexuais humanas, utilizando-se como recurso audiovisual trecho do documentário "The Living Body", na voz do médico Drauzio Varella.

As doenças sexualmente transmissíveis foram o tema seguinte, momento no qual os residentes apresentaram um breve conteúdo sobre doenças como sífilis, HIV, hepatite B, cancro mole e herpes, seguindo-se de dinâmica na qual os alunos eram instigados a identificar as doenças de acordo com os sintomas e sinais descritos em cartazes.

Na oficina sobre Gravidez na Adolescência foi exibido o documentário "Meninas", produzido por Sandra Werneck e Gisela Camara. O longa trata da história de quatro meninas adolescentes de uma comunidade do Rio de Janeiro, que necessitam lidar com os desafios e as responsabilidades de uma gravidez na adolescência. Após a exibição, pôde-se observar a empatia e estranhamento demonstrados pelos adolescentes, principalmente as meninas, a respeito da idade e contexto social das personagens do filme.

As relações familiares foram o assunto do quinto encontro, quando foi exibido o vídeo "O pai está", produzido pela Prefeitura Municipal do Rio de Janeiro. O curta trata do papel dos pais no cuidado com os filhos e as experiências destes no acesso aos serviços de saúde para acompanhamento das crianças. A discussão acerca do tema instigou a reflexão dos jovens sobre a importância da participação do pai no processo de crescimento e desenvolvimento dos filhos.

A sexta oficina abordou os métodos contraceptivos mediante uso de recursos lúdicos como slides, modelos em acrílico, preservativos, cartelas de anticoncepcional, dispositivo intrauterino, dentre outros; propiciando o aprendizado e a conscientização acerca do planejamento familiar. 
O tema do sétimo encontro foi a orientação sexual e a identidade de gênero, em que termos e definições foram esclarecidos e discutidos com os alunos, tendo como referência o guia 'Orientações sobre identidade de gênero: conceitos e termos' ${ }^{20} \mathrm{O}$ debate sobre os estereótipos e preconceitos atribuídos às populações LGBT foi fomentado pela série de vídeos “Não é porque eu sou” do canal 'Põe na Roda' do Youtube, que apresenta depoimentos de homossexuais, bissexuais e transexuais acerca da vivência de suas sexualidades, de maneira a desconstruir estigmas sociais.

No último encontro, abordou-se o tema Projeto de Vida, no qual os estudantes foram estimulados a refletir sobre suas perspectivas de vida, partindo de uma avaliação de sua situações de vida atuais com auxílio do instrumento 'Roda da Vida'. A 'Roda da Vida' consiste em uma ferramenta de coaching, formada por um círculo em forma de gráfico de pizza no qual o indivíduo deve avaliar aspectos de sua vida, como relacionamento amoroso, carreira, trabalho, saúde, social, finanças e família, classificando-os em níveis em uma escala um a dez. ${ }^{21}$ Dessa maneira, os facilitadores promoveram o debate e autorreflexão entre os adolescentes acerca de seus sonhos e desejos para o futuro. Ao findar da atividade, os jovens foram solicitados a avaliar as oficinas por meio de questionário semiestruturado elaborado pelos próprios residentes (Quadro 2).

\section{Oficinas, espaços de aprendizado e construção: a interação entre residentes e adolescentes}

O atendimento à saúde para ser integral necessita aproximar-se das comunidades, ultrapassando os muros das unidades de saúde com estímulo à participação popular. Nesse contexto, a Estratégia de Saúde da Família (ESF) deve integrar-se continuamente com entidades e organizações sociais por meio de parcerias, focando-se nas necessidades e vulnerabilidades apresentadas pelo seu território de atuação. ${ }^{12}$

O cenário escolar apresenta-se como um espaço social privilegiado para o desenvolvimento de ações de educação em saúde. Os adolescentes, a partir de experiências e expectativas de vida, constroem o conhecimento coletivamente despertando a consciência crítica e o autocuidado, tornando-se capazes de compreender suas escolhas, tomar decisões em saúde e atuar como protagonistas nas comunidades em que vivem. ${ }^{11,13,14}$

O Programa Saúde na Escola (PSE), instituído em 2007, tem como objetivo a promoção de ações que visem o enfrentamento de vulnerabilidades que afetam crianças e adolescentes da rede pública de ensino. ${ }^{4} \mathrm{~A}$ implantação do programa possibilita a maior aproximação dos jovens das equipes de ESF e das unidades, assim como, aumento da percepção dos profissionais como promotores de saúde, fomentando atitudes saudáveis entre os jovens. ${ }^{5,12}$

Segundo Martins et al., ${ }^{5}$ as oficinas apresentam abordagem qualitativa, dinâmica e holística, compreendendo o indivíduo em seu ambiente, diante de experiências reveladas próximas a sua realidade, o que possibilita a imersão no contexto sociocultural da comunidade na qual os profissionais estão inseridos. Paralelamente, ao compartilhar informações em um espaço de debate e liberdade de expressão, os estudantes podem compreender sua sexualidade de forma mais saudável e responsável.

O método de oficinas permitiu aos adolescentes expressar seus entendimentos e visões acerca da sexualidade. Observou-se, inicialmente, um certo constrangimento entre os jovens em tratar sobre o tema, porém com o decorrer das atividades e criação de vínculo com os residentes, os estudantes foram 
aumentando sua participação e compartilhamento de experiências pessoais e dúvidas. Ambas as turmas apresentaram comportamentos distintos no decorrer dos encontros, ora participando e interagindo com falas e dúvidas, ora retraídos e dispersos.

Ao se trabalhar com adolescentes, deve-se compreender que estes tendem a rejeitar conceitos e paradigmas apresentados de forma impositiva, dessa forma, deve-se evitar discursos punitivos e amedrontadores acerca dos riscos das DST's, por exemplo. ${ }^{8,18}$ Sendo assim, o diálogo permite aos jovens instigar e mediar esclarecimentos, de maneira horizontal com seus facilitadores/professores, formando vínculos e relações de confiança que dinamizam o processo educativo. ${ }^{8}$

Jeolás e Ferrari ${ }^{17}$ reconhecem que os conhecimentos, apesar de necessários, demonstram-se insuficientes para a adoção de hábitos e práticas sexuais seguras, tornando as oficinas mais relevantes por motivar a autocrítica sobre valores e condutas.

No entanto, vale ressaltar que esses espaços se apresentam como o estímulo inicial de um processo que deve ser complementado pela família, pela escola e por políticas sociais voltadas para os adolescentes, de maneira a desenvolver integralmente a consciência e a corresponsabilização pela sua saúde, tornando-os seres autônomos capazes de realizar escolhas responsáveis sobre a própria vida. ${ }^{14,22}$

\section{Revisitando conceitos e descontruindo tabus: percepções acerca dos adolescentes}

A sexualidade consiste em um componente intrínseco dos seres humanos, englobando aspectos biológicos, psicológicos, culturais e sociais, sendo influenciada intensamente pela sociedade por meio de crenças, tabus, normas e convenções sociais. ${ }^{8}$ Devido ao maior interesse e curiosidade dos jovens sobre o tema, por vezes, os adolescentes acabam recorrendo a fontes de informação não confiáveis; tornando a adolescência um período oportuno para discussões sobre o assunto. ${ }^{2,8}$

Apesar disso, o tabu entorno da sexualidade dificulta a abordagem tanto por parte das famílias, que delegam essa tarefa para a escola, quanto por parte dos professores, que por vezes são impelidos a discutir mesmo sem estarem preparados, além da crença disseminada de que o tema deveria ser tratado em disciplinas como biologia e ciências naturais. ${ }^{8,9}$

As oficinas possibilitaram o compartilhamento de ideias e saberes entre residentes e alunos, contribuindo para o diálogo e reflexões sobre a sexualidade, essenciais ao processo de formação e construção de valores dos indivíduos. Ao serem questionados acerca de conhecimentos prévios sobre métodos contraceptivos, sistemas reprodutores e doenças sexualmente transmissíveis, a maioria dos alunos apresentou conceitos superficiais e de senso comum, com certa dificuldade em comentar determinados assuntos e exprimir opiniões, expectativas e dúvidas, como também identificado em outros estudos. 5,17,23

O Quadro 1 apresenta a percepção dos residentes acerca da receptividade, interação e participação dos estudantes nos encontros, em que se observou padrões de comportamentos e discursos predominantes. Acerca de temas como gravidez na adolescência, violência doméstica e direitos femininos, notou-se que as meninas demonstraram mais proatividade e discursos de emancipação, como na roda de conversa sobre o documentário "Meninas" e do curta "Era uma vez outra Maria", em que foram evidenciadas falas sobre a responsabilização dos homens e mulheres, na prevenção da gravidez; a valorização do papel da mulher na sociedade e igualdade de gênero. 
Quadro 1. Percepções dos residentes facilitadores acerca dos comportamentos dos adolescentes durante as oficinas.

\begin{tabular}{|lll|}
\hline Percepções acerca dos adolescentes & $\mathbf{8}^{\circ}$ ano - matutino & $\mathbf{8}^{\circ}$ ano - vespertino \\
\hline Impressões iniciais e interação com os & $\begin{array}{l}\text { Demonstraram-se interessados e } \\
\text { receptivos. }\end{array}$ & $\begin{array}{l}\text { Demonstraram-se dispersos e agitados. } \\
\text { facilitadores }\end{array}$ \\
& $\begin{array}{l}\text { Coucas interações e dévividas inicialmente. } \\
\text { facilitadores. }\end{array}$ \\
& Meninas: participativas, contribuíam com & Meninas: raramente contribuíam com \\
Participação e contribuições nas rodas de & questionamentos e experiências pessoais. & opiniões, dispersas em certos temas. \\
conversa & Meninos: pouco participativos, & Meninos: participativos, questionavam e \\
& demonstravam timidez ao conversar sobre & contribuíam com experiências pessoais, \\
& os temas, raramente faziam perguntas & de familiares e conhecidos \\
Impressões finais e avaliações dos & Mostraram-se proativos e mais receptivos & Mostraram aumento do interesse e \\
adolescentes & aos facilitadores ao longo das oficinas. & participação ao longo das oficinas. \\
& Avaliações positivas ao término. & Avaliações positivas ao término.
\end{tabular}

Fonte: Elaborado pelos autores.

Ao serem abordados tópicos como identidade de gênero e orientação sexual, os jovens demonstraram certo estranhamento devido ao desconhecimento de determinados termos, bem como curiosidade e interesse sobre o assunto. Não foram identificados discursos ou reações preconceituosas por parte dos adolescentes, o que pode estar relacionado tanto ao grau de conscientização prévia e proporcionado pelas oficinas quanto à presença dos facilitadores.

Em perspectiva comparativa, verifica-se em estudo ${ }^{23}$ realizado em 2005 com adolescentes da instituição Casa da Criança situada no Morro da Penitenciária, mesma comunidade que a Escola Hilda Teodoro, aspectos semelhantes e divergentes no que concerne aos pontos de vista e falas dos jovens.

$\mathrm{Na}$ experiência de Maheirie et al. ${ }^{23}$ evidenciou-se que, apesar de relativizarem relações de gênero, os adolescentes - principalmente as meninas - ainda apresentavam concepções conservadoras sobre os papéis destinados às mulheres na cultura ocidental, quando confrontados com situações mais concretas. Tal comportamento enfatizava o padrão de responsabilização das mulheres sobre a contracepção e a gravidez, confrontando o plano do discurso e as atitudes diante da objetivação concreta. Este padrão de comportamento não foi identificado durante a intervenção na EEB Hilda Teodoro, como enfatizado anteriormente, em que sobressaíram pontos de vista mais progressistas e emancipatórios por parte das adolescentes.

Tais mudanças de paradigmas, tanto por parte das meninas quanto dos meninos, podem expressar as modificações em curso na sociedade brasileira nas últimas décadas, elucidando que o processo de constituição dos sujeitos ocorre por meio de avanços e recuos, ambiguidades e contradições, ${ }^{24}$ que ao longo do tempo podem consolidar novos paradigmas sociais.

\section{Oficinas, fortalezas e limitações: percepções acerca da experiência}

As oficinas de saúde e sexualidade oportunizaram a partilha de conhecimentos e o aprendizado mútuo entre os adolescentes e os residentes de saúde, na medida em que, os jovens profissionais enriqueceram técnica e pessoalmente, e os adolescentes expuseram opiniões e dúvidas desconstruindo tabus e preconceitos.

A intervenção elucidou a importância da articulação estratégica entre a Estratégia de Saúde da Família e a Escola, interação que tem o potencial de contribuir com o maior envolvimento dos profissionais de saúde com a comunidade, ampliando o conhecimento sobre o território de atuação, suas peculiaridades e vulnerabilidades, colaborando para o planejamento estratégico de ações de saúde. 
Entretanto, cabe ressaltar que a proposta desenvolvida também apresentou limitações, como o carater vertical implícito relacionado ao modelo expositivo das oficinas. Ainda que os residentes tenham estimulado a participação e o esclarecimento de dúvidas dos estudantes, os temas e os questionamentos partiram dos facilitadores. Uma possível explicação para a adoção dessa abordagem pode estar associada à própria formação dos profissionais de saúde, que, sujeitos a modelos cartesianos e conteudistas, tendem a reproduzir os paradigmas aos quais foram expostos.

Sugere-se para futuras intervenções a adoção de modelos de ensino mais dialógicos e problematizadores, como na concepção freireana, permitindo aos estudantes alvitrarem temas de acordo com a realidade e necessidades do contexto escolar, desenvolvendo o método de ensino e aprendizagem, baseando-se na construção, criação e recriação do conhecimento. ${ }^{24}$

Apesar dessas limitações, identificaram-se avaliações positivas, por parte dos estudantes, acerca das oficinas, dos recursos utilizados e temas abordados, como observado no Quadro 2. Os temas preferidos pelos adolescentes foram "Projeto de Vida" e "Gravidez na adolescência", e o recurso mais citado foi "vídeos". Algumas sugestões de temas a serem abordados também foram citados, como "trabalho na adolescência" e "pressão familiar", que poderiam ser abordados em futuras intervenções.

Quadro 2. Avaliações dos adolescentes acerca das oficinas por meio de questionário auto-aplicável fornecido pelos residentes facilitadores.

\begin{tabular}{|ccc|}
\hline & Avaliações dos adolescentes acerca das oficinas* & \\
\hline Avaliação final & Recursos preferidos & Temas preferidos \\
\hline Ótimo (13) & Vídeos (17) & Projeto de vida (15) \\
Bom (10) & Rodas de conversa (6) & Gravidez na adolescência (6) \\
Regular (2) & Slides (5) & Métodos contraceptivos (4) \\
Ruim (0) & Dinâmicas (1) & Identidade de gênero e orientação sexual (3) \\
\hline
\end{tabular}

* Tópico avaliado (número de respostas). Fonte: Questionário de avaliação final (*sujeito a perdas devido a ausência de alunos na avaliação).

\section{Conclusão}

As oficinas de saúde e sexualidade desenvolvidas pelos residentes de medicina de família e comunidade e saúde da família representaram uma oportunidade ímpar de interação entre os Programas de Residência em Saúde da Família, as equipes de Estratégia de Saúde da Família e a comunidade, utilizando o cenário escolar como ferramenta para a promoção de saúde e empoderamento social.

Ações comunitárias junto às populações do território têm o potencial de contribuir enormemente com a vivência e formação dos residentes. Espera-se que essa experiência sirva como estímulo para intervenções e trabalhos que visem à saúde dos adolescentes, o debate sobre a sexualidade, assim como, a parceria entre serviços e universidades, tanto na graduação como pós-graduação.

\section{Contribuição dos autores}

Concepção e/ou delineamento do estudo: IGF, MP. Implementação do projeto, organização e execução das oficinas: IGF, MP, DS. Redação preliminar: IGF, MP, DS. Revisão crítica da versão preliminar: IGF, MP, DS.

Todos os autores aprovaram a versão final e concordaram com prestar contas sobre todos os aspectos do trabalho. 


\title{
Conflito de interesses
}

\author{
Declaram não haver.
}

\section{Referências}

1. Pedrosa SC, Costa DVS, Citó MDCO, Luna IT, Pinheiro PNC. Educação em saúde com adolescentes acerca do uso de álcool e outras drogas. Rev Enferm Cent-Oeste Min. 2015;5(1):1535-41.

2. Silva GS, Lourdes LAD, Barroso KDA, Guedes HM. Comportamento sexual de adolescentes escolares. REME Rev Min Enferm. 2015;19(1):154-60. http://dx.doi.org/10.5935/1415-2762.20150013

3. Leite CT, Vieira RP, Machado CA, Quirino GS, Machado MFAS. Prática de educação em saúde percebida por escolares. Cogitare Enferm. 2014;19(1):13-9. http://dx.doi.org/10.5380/ce.v19i1.35925

4. Fonseca FF, Sena RKR, Santos RLA, Dias OV, Costa SM. As vulnerabilidades na infância e adolescência e as políticas públicas brasileiras de intervenção. Rev Paul Pediatr. 2013;31(2):258-64. http://dx.doi.org/10.1590/S0103-05822013000200019

5. Martins CBG, Ferreira LO, Santos PRM, Lopes Sobrinho MW, Weiss MCV, Souza SPS. Oficina sobre sexualidade na adolescência: uma experiência da equipe saúde da família com adolescentes do ensino médio. REME Rev Min Enferm. 2011;15(4):573-8.

6. Brasil. Ministério da Saúde. Secretaria de Atenção em Saúde. Departamento de Ações Programáticas Estratégicas. Diretrizes nacionais para a atenção integral à saúde de adolescentes e jovens na promoção, proteção e recuperação da saúde. Brasília: Ministério da Saúde; 2010.

7. Barros BF, Aurélio EF, Macaya DAM, Marcelo AM, Otto DY, Santos IS. Sexo sem tabu: relato de um caso de ação comunitária, com foco na promoção de saúde de adolescentes, em uma escola técnica na cidade de São Paulo. Rev Med. 2013;92(2):128-33. http://dx.doi. org/10.11606/issn.1679-9836.v92i2p128-133

8. Nothaft SCDS, Zanatta EA, Brumm MLB, Galli KDSB, Erdtmann BK, Buss E, et al. Sexualidade do adolescente no discurso de educadores: possibilidades para práticas educativas. REME Rev Min Enferm. 2014;18(2):284-9.

9. Queiroz AAFLN, Sousa AFLD, Feitosa JJDM, Alves RC, Nery IS, Moura MEB. Sex education for adolescents by teachers from a community education center. Rev Pesqui Cuid Fundam. 2016;8(4):5120-5. http://dx.doi.org/10.9789/2175-5361.2016.v8i4.5120-5125

10. Farias ICV, Sá RMPF, Figueiredo N, Menezes Filho A. Análise da Intersetorialidade no Programa Saúde na Escola. Rev Bras Educ Med. 2016;40(2):261-7. http://dx.doi.org/10.1590/1981-52712015v40n2e02642014

11. Machado MFAS, Gubert FA, Meyer APGFV, Sampaio YPCC, Dias MSA, Almeida AMBAB, et al. The health school programme: a health promotion strategy in primary care in Brazil. J Hum Growth Dev. 2014;25(3):307-12. http://dx.doi.org/10.7322/jhgd.96709

12. Santiago LM, Rodrigues MTP, Oliveira Junior AD, Moreira TMM. Implantação do Programa Saúde na escola em Fortaleza-CE: atuação de equipe da Estratégia Saúde da Família. Rev Bras Enferm. 2012;65(6):1026-9. http://dx.doi.org/10.1590/S0034-71672012000600020

13. Penso MA, Brasil KCTR, Arrais AR, Lordello SR. A relação entre saúde e escola: percepções dos profissionais que trabalham com adolescentes na atenção primária à saúde no Distrito Federal. Saude Soc. 2013;22(2):542-53. http://dx.doi.org/10.1590/S010412902013000200023

14. Machado LDS, Ramos JLS, Machado MFAS, Antão JYFL, Santos SB, Marinho MNASB, et al. Participatory process of health promotion at school. J Hum Growth Dev. 2015;25(3):357-63. http://dx.doi.org/10.7322/jhgd.106014

15. Pinho LMG, Garcia VL, Nogueira-Martins MCF. Implantação da residência médica e multiprofissional em saúde da família em um município paulista: percepção de residentes da primeira turma (2014-2016). Rev Bras Pesqui Saúde. 2017;19(2):106-14.

16. Casanova IA, Batista NA, Ruiz Moreno L. Formação para o trabalho em equipe na residência multiprofissional em saúde. ABCS Health Sci. 2015;40(3):229-33. http://dx.doi.org/10.7322/abcshs.v40i3.800

17. Jeolás LS, Ferrari RAP. Oficinas de prevenção em um serviço de saúde para adolescentes: espaço de reflexão e de conhecimento compartilhado. Ciênc Saúde Coletiva. 2003;8(2):611-20. http://dx.doi.org/10.1590/S1413-81232003000200021 
18. Dias FLA, Silva KL, Vieira NFC, Pinheiro PNC, Maia CC. Riscos e vulnerabilidades relacionados à sexualidade na adolescência. Rev Enferm UERJ. 2010;18(3):456-61.

19. Beserra EP, Sousa LB, Cardoso VP, Alves MDS. Percepção de adolescentes acerca da atividade de vida "exprimir sexualidade". Rev Pesqui Cuid Fundam. 2017;9(2):340-6. http://dx.doi.org/10.9789/2175-5361.2017.v9i2.340-346

20. Jesus JGD. Orientações sobre identidade de gênero: conceitos e termos Guia técnico sobre pessoas transexuais, travestis e demais transgêneros, para formadores de opinião. $2^{\mathrm{a}}$ ed. Brasília; 2012.

21. Torresan ML, Pessotto F, Bueno CH. Mindfulness e coaching: alternativas para o desenvolvimento humano. Rev Carreiras Pessoas. 2018;8(1):130-41. http://dx.doi.org/10.20503/recape.v8i1.33385

22. Salum GDB, Monteiro LAS. Educação em saúde para adolescentes na escola: um relato de experiência. REME Rev Min Enferm. 2015;19(2):246-51. http://dx.doi.org/10.5935/1415-2762.20150039

23. Maheirie K, Urnau LC, Vavassori MB, Orlandi R, Baierle RE. Oficinas sobre sexualidade com adolescentes: um relato de experiência. Psicol Estud. 2005;10(3):537-42. http://dx.doi.org/10.1590/S1413-73722005000300022

24. Miranda ACG, Pazinato MS, Braibante MEF. Temas geradores através de uma abordagem temárica freireana: contribuições para o ensino de ciências. Rev Educ Ciênc Matemática. 2017;7(3):73-92. 